\title{
Analysis of Clinical Efficacy of Xuebijing Combined with Maxing Shigan Decoction in Treating Severe Pneumonia
}

\author{
QIFEI ZHONG, PING TANG, ZONGLI SUN AND HUAIGANG XU*
}

Department of Critical Medicine, Shuyang Hospital of Traditional Chinese Medicine, Suqian, Jiangsu Province 223600, China

Zhong et al.: Effect of Xuebijing and Maxing Shigan Decoction in Treating Severe Pneumonia

\begin{abstract}
This research aims to discuss the clinical efficacy of Xuebijing joint Maxing Shigan decoction treatment in severe pneumonia patients, in the meantime to discuss the effects of drugs combination on inflammation indicators, arterial blood gases and clinical safety. We chose 120 patients with severe pneumonia in our hospital as the study cohort. Divided them into control group $(n=60)$ and observation group $(n=60)$. Control group received antibiotics, observation group received antibiotics as well but combined with Xuebijing and Maxing Shigan decoction. Compared both groups in clinical effective rate, inflammation index, arterial blood gas and the incidence of drug related complications after treatment. After treatment, observation group's inflammation indexes (C-reactive protein, procalcitonin), arterial blood gas changes (partial pressure of oxygen, partial pressure of carbon dioxide) and traditional Chinese medicine syndrome scores were significantly reduced $(\mathbf{p}<\mathbf{0 . 0 5})$. The clinical efficacy of observation group was higher than the other $(p<0.05)$, it possessed no remarkable difference in both groups complications rate $(p>0.05)$ and it was of no statistical difference. Xuebijing combined with Maxing Shigan decoction can effectively treat patients with severe pneumonia. The clinical symptoms are significantly improved, and inflammation indicators and arterial blood gas conditions can be improved without increasing the adverse incidence and complications of drugs. The combined use is worthwhile for patients with severe pneumonia.
\end{abstract}

Key words: Xuebijing, Maxing Shigan decoction, severe pneumonia, clinical efficacy

Severe pneumonia is a critical type of respiratory disease, which can be complicated by severe symptoms or other serious complications of the system and it has the characteristics of rapid progress and high fatality rate ${ }^{[1]}$. The main severe pneumonia signs were respiratory failure; there is often accompanied systemic inflammation, for example, multiple organ failure, septic shock, diffuse intravascular coagulation, etc., Clinical performances such as palpitations, dyspnea, hypotension, high fever, shock and consciousness disorder, etc., occurs frequently ${ }^{[2,3]} .95 .20 \%$ of severe pneumonia patients with detected pathogenic microorganisms, of which bacterial infection accounted for $49.2 \%$. Therefore, we must use antibiotics ${ }^{[4]}$. An early effective anti-infection works on reducing severe pneumonia patients mortality ${ }^{[5]}$. As antibiotics use wide spreading, great changes have taken place in pathogenic bacteria. Bacterial resistance has become a global problem $^{[6]}$. At the same time, the serious severe pneumonia complications such as sepsis, empyema, pericarditis, respiratory distress syndrome, multifunctional organ failure, etc. caused continuous deaths ${ }^{[7,8]}$. At present, conventional therapy is ineffective and we can mainly adopt symptomatic treatment to cure severe pneumonia ${ }^{[9]}$. Under these circumstances, combination medication becomes more and more important, Traditional Chinese Medicine (TCM) has unique advantages on severe pneumonia treatment. In recent years, Chinese medical workers have adopted Xuebijing injection, which has the effect of improving blood circulation and dispersing stasis, to treat severe pneumonia $^{[10,11]}$ and made a good clinical effect. We can find lots of publications of systematic reviews of Xuebijing about severe pneumonia treatment in Chinese journals in 2012, 2014 and 2015, and all of them have approved that Xuebijing is effective on severe pneumonia treatment ${ }^{[12]}$. However, it is a controversial topic whether Xuebijing works on reducing severe pneumonia patient's mortality. Maxing Shigan decoction is a TCM commonly used in severe

*Address for correspondence

E-mail: xutenye@126.com 
pneumonia treatment these years. It is proved to be effective on clearing heat and detoxification, reducing phlegm, anti-infection etc., ${ }^{[13]}$. The combination of Xuebijing and Maxing Shigan decoction has significant effect on severe pneumonia treatment. So as to further understand the combination clinical efficacy, we made this research to obtain more references for clinical applications. The following study you can find out the specific investigation report. We chose 129 patients with severe pneumonia in our hospital from February 2016 to March 2021 as the study cohort. Divided them into treatment group and control group, with 60 cases in each group. The general information of both groups is displayed in Table 1. We had already got approval from Medical Ethics Committee of our hospital. Meet the diagnostic criteria proposed in "Chinese Expert Consensus on Clinical Practice of Severe Pneumonia"[14] and the pathogenic examinations of pneumonia are all bacterial pneumonia; No allergies to Xuebijing and Maxing Shigan decoction in this study; all patients obtained informed consent for treatment. The patient also suffers from severe cardiopulmonary dysfunction and other diseases; Women who are pregnant, breastfeeding, planning to become pregnant or women with reproductive potential who do not use recognized contraceptive methods; Those who are allergic to Xuebijing or Maxing Shigan decoction; Those who suffer from other chronic lung diseases such as active tuberculosis, bronchiectasis, cystic fibrosis or active lung cancer; Patients have diseases that threaten life or seriously exist, it is on a unstable state, such as myocardial infarction; Patients who is confirmed renal insufficiency or suspected/creatinine clearance rate $<40$ ml/min; Patients whose Alanine Aminotransferase (ALT), Aspartate Aminotransferase (AST) or alkaline phosphatase levels exceed 3 times upper limit normal limit; Patients who has epilepsy record, myasthenia gravis symptom, alcoholism, heavy smoking custom (exceed 40 cigarettes per day) and drug addiction. Both groups were given the same antibiotic treatment after admission and then according to the results of drug susceptibility, the corresponding antibiotic treatment was given. At the same time, fluid and nutritional treatments were given according to the results of the internal environment examination. For patients with respiratory failure, auxiliary treatment with ventilator was given when necessary. Treated observation group the same as the other group, then adopted Maxing Shigan decoction and Xuebijing. Ephedra $15 \mathrm{~g}$, almond $15 \mathrm{~g}$, licorice $12 \mathrm{~g}$, gypsum $20 \mathrm{~g}$; 1 dose per day, orally after decoction, nasal feeding is given to those who are seriously ill and cannot be taken orally, once in the morning and once in the evening. Xuebijing injection $50 \mathrm{ml}+$ sodium chloride $100 \mathrm{ml}$, intravenous drip for every $12 \mathrm{~h}(\mathrm{Q} 12 \mathrm{H})$. The treatment cycle of the two groups was $2 \mathrm{w}$. The clinical efficacy means the proportion of patients whose symptoms or signs subside or improve after $7 \mathrm{~d}$ treatment and without requiring re-hospitalization. The clinical efficacy evaluation indicators include: clinical symptoms and signs; imaging examination results; inflammatory markers, such as Procalcitonin (PCT) or C-Reactive Protein (CRP) level. Clinical recovery means that all three indicators have improved to the normal range; Markedly effective means that all three indicators have been significantly improved; Effective means that two of the evaluation indicators have been significantly improved; Clinical ineffectiveness is defined as one of the following conditions: Symptoms or signs getting deteriorated or persisted or needed other treatments during treatment; $3 \mathrm{~d}$ treatment later, severe pneumonia caused death; Related pneumonia complications appears, such as empyema or lung abscess. Patients with uncertain clinical efficacy are such situations below: Patients required to be transferred to other hospital or they refused further treatment; Died within $2 \mathrm{~d}$ because of pneumonia after treatment or died because of other reasons except pneumonia during treatment; Due to allergies, serious adverse events or other personal reasons lead to incomplete treatment. Arterial blood gas changes (Partial Pressure of Oxygen $\left(\mathrm{PaO}_{2}\right)$, Partial Pressure of Carbon Dioxide $\left(\mathrm{PaCO}_{2}\right)$ ), inflammation indicators (PCT, CRP), traditional Chinese medicine syndrome scores, risk of adverse events (nausea and vomiting, diarrhea, constipation, blood pressure fluctuations, skin rashes, liver and kidney abnormalities), etc. We adopt Statistical Package for the Social Sciences (SPSS) 26.0 to analyze data, use $\mathrm{n} \%$ to express technical data and use $\chi^{2}$ to test. Use mean \pm standard deviation to indicate measurement data that is accord with normal distribution and homogeneity of variance, compare both groups by independent sample $t$ test, and before and after treatment within the same group comparison was expressed by paired t test. Use median and interquartile range to show data that did not accord with normal distribution; the Wilcoxon test was used for group comparison. $\mathrm{p}<0.05$ was supported to possess statistical significance. There was not any significant difference between two groups in terms of age, gender, basic diseases, etc., and it possessed no statistical significance $(\mathrm{p}>0.05)$. These two groups possessed comparability. See the following 
Table 1. There was not any significant differences in TCM syndrome scores or inflammation indexes (CPR, PCT) between both groups and there was no statistical significance $(\mathrm{p}>0.05) ; 2 \mathrm{w}$ treatment later, both groups TCM syndrome scores and inflammation indexes were significantly lower contrast before treatment $(p<0.05)$; See the following Table 2 . There was zero difference in the arterial blood gas analysis $\mathrm{PaO}_{2}$ and $\mathrm{PaCO}_{2}$ levels of both groups before treatment, it possessed no statistical significance ( $\mathrm{p}>0.05)$; After $2 \mathrm{w}$ treatment, the arterial blood gas analysis $\mathrm{PaO}_{2}$ and $\mathrm{PaCO}_{2}$ levels of the two groups were remarkably different, it had statistical significance $(\mathrm{p}<0.05)$, see below Table 3 for details. In the observation group, 12 cases $(20 \%)$ had adverse reactions, among them there were 3 cases blood pressure fluctuation, 3 cases skin rash, 2 cases nausea and vomiting, 3 cases diarrhea and constipation and 1 case of liver and kidney abnormalities. Adverse reactions occurred in 14 cases $(23.3 \%)$ in the other group, among them 2 cases blood pressure fluctuations, 4 cases skin rash, 3 cases nausea and vomiting, 4 cases diarrhea and constipation and 1 case liver and kidney abnormalities. Adverse drug reactions between both groups have no statistical difference $(p>0.05)$. Both groups adverse drug reactions incidence during treatment were almost the same and there was no statistical significance ( $p>0.05)$. Let's see the following Table 4. Observation group total clinical efficacy rate was remarkably higher than the other $(\mathrm{p}<0.05)$, see the following Table 4. Among types of critical diseases, severe pneumonia is the most common in clinic. It often occurs in elderly or children, has the feature of rapid onset, fast illness changes and high mortality. Severe pneumonia mainly causes death in intensive care unit or respiratory department ${ }^{[15]}$. Severe pneumonia is often caused by pathogenic bacteria infections. Unreasonable antibiotics use and patient's physical condition will gradually make lung infections aggravated, then it will damage multiple organs and worsen to severe pneumonia ${ }^{[16]}$. It belongs to the field of "wind-warm lung-heat disease", "pulmonary fever disease" and "pulmonary carbuncle" in TCM. The TCM believes that severe pneumonia is caused by insufficient vitality or mistreatment, the intrusion of wind and heat, and severe pneumonia etiology and pathogenesis may be excessive toxic heat ${ }^{[14-17]}$. Pathogenic bacteria spreading, environmental pollution and people's living and eating habits lead to pneumonia increasing year by year. A large amount of clinical practices have proved that severe pneumonia conventional antibiotics had less effect contrast TCM combined with antibiotics ${ }^{[18]}$.
Xuebijing injection has a widely application in recent years. It is composed of safflower, red peony root, Chuanxiong, Salvia miltiorrhiza and Angelica, among which red peony root has the functions of clearing heat and cooling blood, dispersing blood stasis and relieving pain. Ligusticum chuanxiong has the effects of invigorating blood, promoting gas, dispelling wind and relieving pain ${ }^{[19]}$. Many studies have approved that Xuebijing injection joint conventional antibiotics have better effect on severe pneumonia treatment. Maxing Shigan decoction is composed of ephedra, almonds, licorice and gypsum. It is mainly used to treat exogenous wind invasion and pathogenic heat blocking the lung ${ }^{[20]}$. Experiments show that Xuebijing injection and Maxing Shigan decoction have a strong inhibitory effect on pathogenic bacteria such as Streptococcus pneumoniae, type B hemolytic Streptococcus and Staphylococcus aureus. Therefore, this study aims to discuss the clinical efficacy of Xuebijing injection and Maxing Shigan decoction in severe pneumonia, and to provide practical, objective and powerful evidence for clinical diagnosis and treatment. TCM thought pneumonia belongs to the field of asthma and cough, and the symptoms such as cough, sputum, fever and asthma should be treated; other symptoms caused by exogenous infection into the lungs should be mainly treated by relieving fever and soothing the lungs ${ }^{[17]}$. Modern pharmacology indicates that Qi deficiency drugs have multiple effects on antipyretic and analgesic, antiviral and antibacterial symptoms. For example, Tanreqing's main drug components are Scutellaria, bear gall powder, dogwood, honeysuckle and forsythia, which have the effects of clearing away heat and detoxifying. It also has a significant relieving effect on fever, cough and sore throat caused by lung diseases, which has been approved by a large amount of studies, but it does not have the ability of anti-inflammatory and immune regulation ${ }^{[21]}$. At the same time, a large number of studies have proved that Xuebijing and Maxing Shigan decoction have great clinical value in severe pneumonia, but there is no clinical application of the combined use of the two drugs. The combination of Xuebijing injection and Maxing Shigan decoction in our study can remarkably improve the clinical efficacy of severe pneumonia without increasing the incidence of adverse drug reactions, which is the same as the results of previous studies. However, this study has some shortcomings. Divided clinical subjects randomly into groups and double-blind enrollment were not achieved. More confounding factors interfered with this study to a certain extent, such as whether there are infections in 
other parts, blood pressure, blood sugar and other factors. It may affect the results of this research topic and needs further improvement. In summary, Xuebijing combined with Maxing Shigan decoction can effectively treat patients with severe pneumonia. It has obvious clinical value in improving clinical efficacy and improving inflammatory indicators and arterial blood gases, and does not increase the incidence of adverse drugs. It can be used in clinic.

\section{Conflict of interests:}

The authors declared no conflicts of interest.

TABLE 1: COMPARISON OF BOTH GROUPS GENERAL INFORMATION

\begin{tabular}{lcccc}
\hline & $\begin{array}{c}\text { Observation group } \\
(\mathrm{n}=60)\end{array}$ & Control group $(\mathrm{n}=60)$ & $\mathrm{t} / \chi^{2}$ & $\mathrm{p}$ \\
\hline Age & $59.07 \pm 12.73$ & $60.5 \pm 12.55$ & -0.62 & 0.55 \\
Female & $36(60 \%)$ & $35(58.3 \%)$ & 0.07 & 0.79 \\
Complications & & & 0.44 & 0.5 \\
Hypertension & $10(16.7 \%)$ & $11(18.3 \%)$ & 0.12 & 0.734 \\
Diabetes & $8(13.3 \%)$ & $9(15.0 \%)$ & 0.32 & 0.57 \\
Other & $8(13.3 \%)$ & $10(16.7 \%)$ & 0.3 & 0.58 \\
\hline
\end{tabular}

TABLE 2: COMPARISON OF BOTH GROUPS TCM SYNDROME SCORES AND INFLAMMATION INDEXES

\begin{tabular}{lccccccc}
\hline \multirow{2}{*}{ Grouping } & $\begin{array}{c}\text { Number of } \\
\text { cases }\end{array}$ & \multicolumn{2}{c}{ TCM syndrome score } & \multicolumn{2}{c}{ CRP $(\mathrm{mg} / \mathrm{l})$} & \multicolumn{2}{c}{ PCT (ng/ml) } \\
\cline { 3 - 7 } & $\begin{array}{c}\text { Before } \\
\text { treatment }\end{array}$ & $\begin{array}{c}\text { After } \\
\text { treatment }\end{array}$ & $\begin{array}{c}\text { Before } \\
\text { treatment }\end{array}$ & $\begin{array}{c}\text { After } \\
\text { treatment }\end{array}$ & $\begin{array}{c}\text { Before } \\
\text { treatment }\end{array}$ & $\begin{array}{c}\text { After } \\
\text { treatment }\end{array}$ \\
\hline $\begin{array}{l}\text { Observation } \\
\text { group }\end{array}$ & 60 & $25.75 \pm 6.2$ & $13.56 \pm 2.84$ & $119.6 \pm 25.8$ & $51.25 \pm 15.1$ & $2.20 \pm 1.90$ & $0.34 \pm 0.20$ \\
Control group & 60 & $25.61 \pm 6.3$ & $15.9 \pm 4.34$ & $120.5 \pm 22.3$ & $60.42 \pm 21.08$ & $2.3 \pm 1.62$ & $0.48 \pm 0.36$ \\
$\mathrm{t}$ & & 0.123 & -3.53 & -0.20 & -2.73 & -0.31 & -2.63 \\
$\mathrm{p}$ & & 0.91 & 0.006 & 0.84 & 0.006 & 0.75 & 0.006 \\
\hline
\end{tabular}

Note: TCM: Traditional Chinese Medicine; CRP: C-Reactive Protein and PDT: Procalcitonin

TABLE 3: COMPARISON OF BOTH GROUPS $\mathrm{PaO}_{2}$ AND PaCO ${ }_{2}$ LEVELS OF ARTERIAL BLOOD GAS BEFORE AND AFTER TREATMENT

\begin{tabular}{lccccc}
\hline \multirow{2}{*}{ Grouping } & \multirow{2}{*}{ Number of cases } & \multicolumn{2}{c}{$\mathrm{PaO}_{2}$} & \multicolumn{2}{c}{$\mathrm{PaCO}_{2}$} \\
\cline { 3 - 6 } & & Before treatment & After treatment & Before treatment & After treatment \\
\hline Observation group & 60 & $43.4 \pm 14.03$ & $78.8 \pm 23.98$ & $68.18 \pm 22.9$ & $48.98 \pm 14.68$ \\
Control group & 60 & $44.4 \pm 13.64$ & $59.4 \pm 12.4$ & $65.05 \pm 17.07$ & $56.86 \pm 13.03$ \\
$\mathrm{t}$ & & -0.39 & 5.56 & 0.84 & -3.10 \\
$\mathrm{p}$ & & 0.69 & 0.000 & 0.39 & 0.002 \\
\hline
\end{tabular}

TABLE 4: COMPARISON OF BOTH GROUPS ADVERSE REACTIONS DURING TREATMENT

\begin{tabular}{|c|c|c|c|c|c|c|c|}
\hline Grouping & $\begin{array}{c}\text { Number of } \\
\text { cases }\end{array}$ & $\begin{array}{l}\text { Nausea and } \\
\text { vomiting }\end{array}$ & $\begin{array}{l}\text { Diarrhea and } \\
\text { constipation }\end{array}$ & $\begin{array}{l}\text { Blood } \\
\text { pressure } \\
\text { fluctuation }\end{array}$ & Skin rash & $\begin{array}{c}\text { Liver and } \\
\text { kidney } \\
\text { abnormalities }\end{array}$ & Incidence \\
\hline $\begin{array}{l}\text { Observation } \\
\text { group }\end{array}$ & 60 & 2 (3.33\%) & $3(5 \%)$ & $3(5 \%)$ & $3(5 \%)$ & $1(1.66 \%)$ & $12(20 \%)$ \\
\hline Control group & 60 & $3(5 \%)$ & $4(6.66 \%)$ & $2(3.33 \%)$ & $4(6.66 \%)$ & 1 (1.66 \%) & $14(23.3 \%)$ \\
\hline $\mathrm{t}$ & & & & & & & 0.2 \\
\hline$P$ & & & & & & & 0.65 \\
\hline
\end{tabular}




\section{REFERENCES}

1. Mizgerd JP. Pathogenesis of severe pneumonia-advances and knowledge gaps. Curr Opin Pulm Med 2017;23(3):193-7.

2. Krutikov M, Rahman A, Tiberi S. Necrotizing pneumonia (aetiology, clinical features and management). Curr Opin Pulm Med 2019;25(3):225-32.

3. Bartoš H, Džupová O. Severe community-acquired pneumonia in intensive care. Epidemiol Mikrobiol Imunol 2020;69(4):15963.

4. De Pascale G, Bello G, Tumbarello M, Antonelli M. Severe pneumonia in intensive care: Cause, diagnosis, treatment and management: a review of the literature. Curr Opin Pulm Med 2012;18(3):213-21.

5. De Pascale G, Bello G, Antonelli M. Steroids in severe pneumonia: A literature review. Minerva Anestesiol 2011;77(9):902-10.

6. Quinton LJ, Walkey AJ, Mizgerd JP. Integrative physiology of pneumonia. Physiol Rev 2018;98(3):1417-1464.

7. Jia M, Huang W, Li L, Xu Z, Wu L. Statins reduce mortality after non-severe but not after severe pneumonia: A systematic review and meta-analysis. J Pharm Pharm Sci 2015;18(3):286302.

8. Corrales-Medina VF, Musher DM, Shachkina S, Chirinos JA. Acute pneumonia and the cardiovascular system. Lancet 2013;381(9865):496-505.

9. de Benedictis FM, Kerem E, Chang AB, Colin AA, Zar HJ, Bush A. Complicated pneumonia in children. Lancet 2020;396(10253):786-98.

10. Jingsong L. The effect of Xuebijing injection in the treatment of severe pneumonia and its influence on patient's blood gas indexes. Contemporary Med Forum 2021;12(19).

11. Hang R. To investigate the effect of Xuebijing combined with fiberoptic bronchoscope sputum suction and lavage on the level of serum inflammatory factors in patients with severe pneumonia. Chin Health Care Nutr 2021;6(31).

12. Qiu S. Effect of Xuebijing injection on inflammatory factor of patients with severe pneumonia. Chin Foreign Med Treat $2015 ; 28(34)$.
13. Yi Z, Xiaolin F, Bo L. Clinical curative effect of modified Maxing Shigan decoction on severe pneumonia and its effects on pulmonary function and the level of HMGB1. World Chin Med 2018;9(13).

14. Emergency Physicians Branch of Chinese Medical Association. Expert consensus on clinical practice of emergency severe pneumonia in china. Chin J Crit Care Med 2016;2(36).

15. Póvoa P, Coelho L, Salluh J. When should we use corticosteroids in severe community-acquired pneumonia? Curr Opin Infect Dis 2021;34(2):169-74.

16. Jouan Y, Si-Tahar M, Paget C. Severe pneumonia and acute respiratory distress syndrome: Implication of unconventional T cells. Rev Mal Respir 2019;36(4):451-4.

17. Lu Y, Ding J, Liu Q. Research progress on immune imbalance mechanism and traditional Chinese medicine treatment of severe pneumonia. Med Pharm J Chin People's Liberation Army 2017;4(29).

18. You W, Zhou L. Treatment progress of Chinese medicine in severe pneumonia. Shaanxi J Tradit Chin Med 2021;4(42).

19. Li Y, Zhu H. Evaluation on effect in infectious pneumonia of intervention treatment with Maxing Shigan mixture. AntiInfect Pharm 2012;3(9).

20. Xu G. Observation on the curative effect of Maxing Shigan decoction combined with Qianjin Weigan decoction in the treatment of severe pneumonia. J Emerg Tradit Chin Med 2016;9(25).

21. Zhang H. Clinical Study of Tanreqing injection combined with piperacillin sulbactam in the treatment of senile severe pneumonia. Res Integr Tradit Chin West Med 2021;1(13).

This is an open access article distributed under the terms of the Creative Commons Attribution-NonCommercial-ShareAlike 3.0 License, which allows others to remix, tweak, and build upon the work non-commercially, as long as the author is credited and the new creations are licensed under the identical terms

This article was originally published in a special issue, "Novel Therapeutic Approaches in Biomedicine and Pharmaceutical Sciences" Indian J Pharm Sci 2021:83(6) Spl Issue "135-139" 